\title{
Quantitative Evaluation for Structure Difference between Central Region and Periphery in Al-Al Explosive Welding Interface
}

\author{
Fu Yanshu, Xia Meng, Wang Zhen \\ Nanchang University, Nanchang 330031, China
}

\begin{abstract}
Because of lateral sparse wave, there are structure differences between central region and periphery in explosive welding interface. However, academic estimations for the difference of interfaces morphology are still in the qualitative level, and quantitative analysis methods have not been established. This paper tries to evaluate the difference of explosive welding interface morphology quantitatively. First of all, an elastic-viscous model was established to analyze the forming of wavy interface, and symmetrical $2024 \mathrm{Al}$ explosive welding was carried out for testing. Then the interface images of both central district and periphery were detected by three-dimensional ultra-depth microscopy, and a demarcation line for the central area and periphery was defined based on the impact impulse. Furthermore, the fractal dimensions and multi-fractal spectra of the images were calculated on the basis of fractal theory. According to the physics meaning of fractal characters of images, the quantitative descriptions have been achieved for explosive welding interface structure difference.
\end{abstract}

Key words: explosive welding; interface morphology; fractal dimensions; quantitative characterization

With the development of industry, people have higher requirements for material properties and scale. Composite plates produced by explosive welding with good corrosion resistance and mechanical properties can be used in chemical, nuclear and pressure vessels industries. As a technique which can produce large area bonding between metal plates, explosive welding has been deeply researched and extensively applied in engineering. There are many enterprises specializing in metal plate explosive welding and forming a large potential industry in China.

Researchers find that welding interfaces have complex wavy structure and generally believed that the interface structure directly is related to the quality of welding products. Therefore researching and understanding for explosive welding interface structure have profound scientific significance. It has sparked researchers to carry out experimental observation, theoretical analyses and numerical calculations extensively.
Experimental results show that the Kelvin-Helmholtz instability leads the wave to lose symmetry and forms crests shape interface ${ }^{[1]}$. Both wave length and amplitude are associated with to flyer plate thickness, flyer impact velocity and energy density. Shapes of the waves in the welding interface depend on the flyer plate and fall roughly into three classes, straight, smooth-wavy and wavy with some vortex shedding ${ }^{[1-6]}$. Furthermore, calculations reveal that perturbations develop only in the presence of a technological microgap of several tens of micrometers between the metal layers, and yield a spectrum of perturbation wavelengths ${ }^{[2]}$.

Research results have revealed that explosive welding interfaces morphologies are influenced by several factors and show asymmetrical structure. However, most of them are subjective approaches based on experimental results, and no adequate conclusions have described the interface structure quantitatively and been accepted commonly. Thus, it is necessary to establish a method to measure and estimate

Received date: July 15, 2016

Foundation item: National Natural Science Foundation of China (11202093, 51465039)

Corresponding author: Fu Yanshu, Ph. D., Associate Professor, School of Mechanical and Electrical Engineering, Nanchang University, Nanchang 330031, P. R. China, Tel: 0086-791-83969635, E-mail: yshfu@ncu.edu.cn 
interface structure quantitatively.

\section{Theory Model for Wave Instability and Pertur- bations}

During explosive welding, high velocity impact between flyer and basic plate generating a dynamic pulse leads to thermal softening of the fly plate, where Kelvin-Helmholtz instability occurs $^{[4]}$. Additionally, interface waves form only at the impact zone and its vicinity, Since metal flow is induced in this elevated temperature and high pressure region ${ }^{[7]}$.

Based on the instability and perturbation model, a simplified fluid-plastic model was established on Fig.1 to research the welding interface structure. In this model, it is assumed that there is a thin fluid film whose thickness is $h$ on the reverse side surface of the fly plate, and its speed is $u_{0}$ along the tangential of plate, and the film has some small normal perturbations $\vec{n}$ within certain frequencies. Therefore the film slides in tangential direction and vibrates in normal simultaneously.

According to the model shown in Fig.1, activated by the detonation wave, the metal performs as elastic viscous-plastic fluid. It is a common belief that the metal is incompressible, and the stress tensor can be written:

$$
\sigma_{i k}=-p \delta_{i k}+G\left(\frac{\partial u_{i}}{\partial x_{k}}+\frac{\partial u_{k}}{\partial x_{i}}\right)+\mu\left(\frac{\partial \dot{u}_{i}}{\partial x_{k}}+\frac{\partial \dot{u}_{k}}{\partial x_{i}}\right)
$$

where $\sigma_{i k}$ is the stress tensor, $u_{i}$ is a component of the displacement vector $u$, the time derivative of $u$ is the flow speed, symbolized as $\dot{u}$. The first term on the right-hand side in Eq.(1) is the fluid pressure $p$, the second and third terms are the elastic stress and the viscous stress, respectively, while $G$ and $\mu$ are the shear modulus and the dynamic viscosity coefficient of the medium respectively.

The corresponding momentum equation of Eq.(1) can be written:

$$
\rho \ddot{u}+\nabla p=G \Delta u+\mu \Delta \dot{u}+\rho g
$$

From Fig.1, the flying plate performing as fluid state is in touch with and above the air, and thus the density of upper fluid larger than that of lower fluid which is the formation condition of Rayleigh-Taylor instability is satisfied. Besides, the film slides along the basic fluid with a constant speed $u_{0}$,

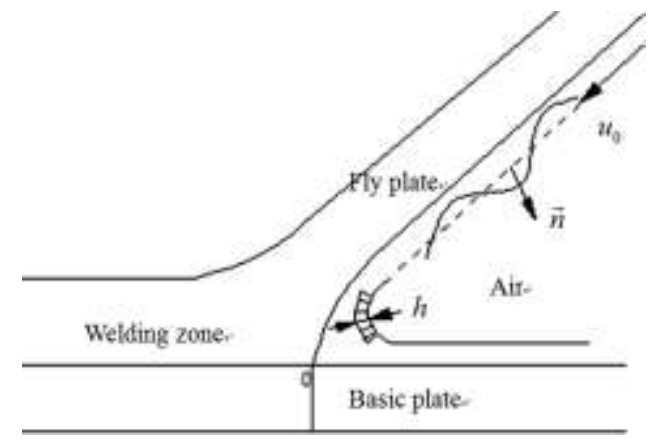

Fig.1 Fluid-plastic model of explosive welding (EXW) resulting in a velocity difference along the tangential between the film and the air, which is the formation condition of Kelvin-Helmholtz instability. These two conditions inevitably lead to instability of fluid film. But in Eq.(2), there are elastic and viscous stresses on metal flow film which will truncate the high frequency oscillations and the further development of the instability ${ }^{[8,9]}$; thereby the instability can not develop infinitely. The competition between tension stresses and instabilities varies with elastic-viscous properties, metal film velocity and surface tension, etc. Whilst, these factors directly relate to local detonation pressure, fluid film temperature and impact velocity. Hence it is difficult to measure the competition on interface structure timely. Fortunately, we only pay attention to the interface after welding, and the competition during welding is not what we care about.

Welding interface profiles are usually rough and have more intensive microscope structures. But how shall we measure it? It was not until the time of Benoit Mandelbrot, however, that dramatic progress was made. Benoit Mandelbrot established the fractal geometry which was characterized by the properties of continuity, nondifferentiability, scale invariance, and self-affinity ${ }^{[10]}$. The fractal geometry is successful to describe disordered phenomena in various fields of science and engineering, and thus it is naturally suitable for explosive welding interface description too.

\section{Experiment}

For the sake of testing, symmetric explosion welding experiments were launched with two layers of homogeneous 2024 Al plates. In order to separate the welding interface conveniently without damaging it, the combination surfaces of flying and basic plate were not carefully polished and cleaned before welding. After welding, the weldment was slowly torn along the detonation direction by a mechanics device, and then cut into testing samples. Besides, samples were soaked in acetone solvent for $2 \mathrm{~h}$ and washed by ultrasonic wave before observing. The wavy interfaces are shown in Fig.2. Most black areas of the interface are the ablation traces of the residual oil contamination. Therefore, it is reasonable to believe that the sample shown in Fig.2 is an explosive welding interface, but not fracture surface.

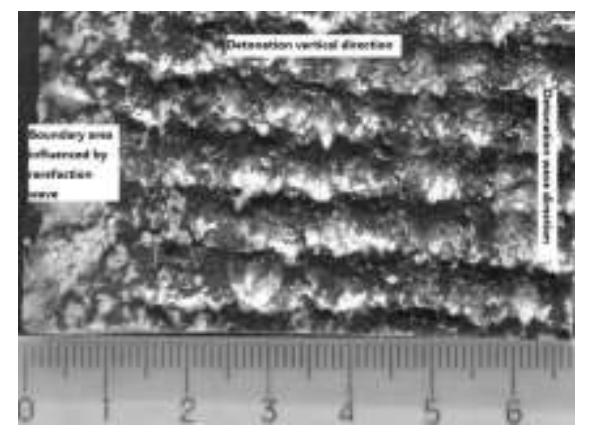

Fig.2 Morphology of welding interface 
Examining the whole morphology of the sample shown in Fig.2, it is evident that characters of concave-convex are relatively regular. But with the magnification increasing, the concave-convex characters of each partial region are different and indicate the morphology has a more intricate structure. In addition, compared with the central region, the periphery which is activated by rarefaction wave during welding process has a relatively flat structure.

\section{Results}

Several regions of the sample shown in Fig.2 are observed by the three-dimensional super-depth microscope and the morphology images of both the central and the periphery regions are shown in Fig.3.

Fig.3a reveals the central region peaks and valleys features of Fig.2 apparently, while in Fig.3b topography undulation is not remarkable. The reason is that the lateral rarefaction wave quickly acts on the boundary area, and then leads to unloading and smaller pulse of detonation pressure acting on the boundary area than that in the central area of the welding interface. According to Ref. [11] which suggested the pressure deforming pulse $I_{\mathrm{d}}$ to describe interface deformation, the pulse in the general case can be calculated by the equation:

$$
I_{\mathrm{d}}=\int_{0}^{\tau_{\mathrm{w}}} p(\tau) \mathrm{d} \tau=\int_{0}^{\tau_{\mathrm{w}}} p_{\max } \mathrm{e}^{(\tau / \theta)} \mathrm{d} \tau
$$

In equation (3), parameters physics meaning are the same as the explanation of Ref. [11], where $p_{\max }$ is the peak pressure in the collision point, $\tau_{\mathrm{w}}$ is the plastic deformation duration (or welding time) after the collision point, and $\theta$ is a time constant describing the pressure decrease gradient in the joint zone. The $I_{\mathrm{d}}$ integral parameter properly determines the energy

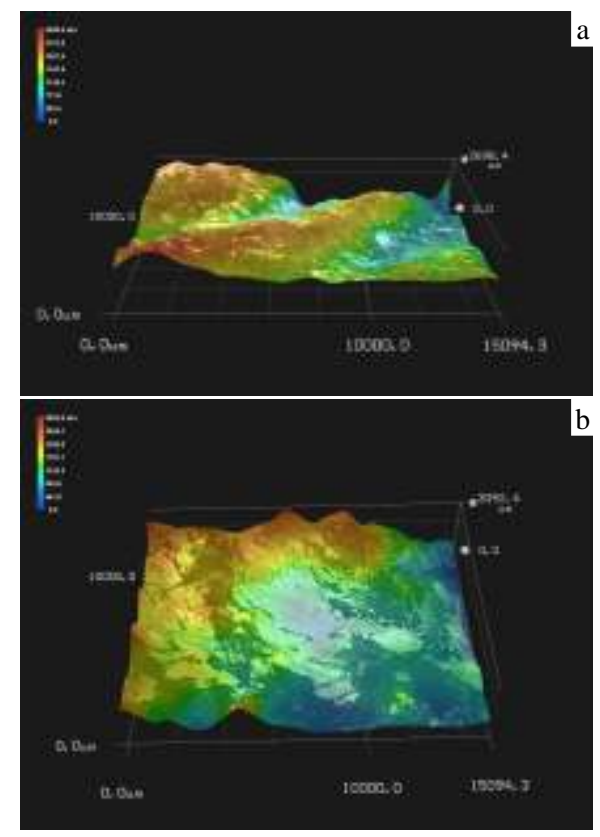

Fig.3 Morphologies of central region(a) and periphery (b) of explosive welding (EXW) interface conditions of joint formation at pressure, which affects the near-contact layers of the joint within some time period and performs certain work on metal plastic deformations in the layers. It should be noted that as the pressure increases and the applied time increases, the portion of energy spending on the plastic deformation of metal in the welding zone increases, which finally achieves the system energy balance.

Ignoring the influence of the lateral rarefaction on the chemical reactions of the explosive detonation wave, the explosive detonation pressure is equal regardless of whether in periphery or in central region. Then the collision pressure in welding points all over the plate is the same as $p_{\max }$, but only the plastic deformation duration $\tau_{\mathrm{w}}$ is different. The duration can be estimated by the lateral scattering theory of detonation products. As shown in Fig.4, explosive thickness is $H$, the angle between oblique detonation wave front $O A B C$ and upper surface $O E$ of fly plate is $\varphi_{0}$, and the detonation wave spreads in normal direction with a constant speed $D$. According to the detonation theory, in Fig.4, products behind detonation wave will firstly develop to central rarefaction wave which is the Prandtl-Meyer (P-M) flow area, and then products scatter out from the P-M boundary. The scattering speed of the product at element $A$ from P-M boundary can be resolved into three components, the normal component along $\vec{n}$, the first tangential component along $\vec{\tau}_{1}$ and the second component along $\vec{\tau}_{2}$. When considering the influence of the rarefaction wave on explosive welding interface, scattering characteristics of the normal direction are nearly the same at everywhere. Thus by comparing with the scattering characteristics of the products at four key points $O, A, A_{1}$, and $A_{2}$ in Fig.4, we can know:

(1) At point $O$, the rarefaction wave along $\vec{\tau}_{2}$ arrives at it later than that along $\vec{\tau}_{1}$. Therefore the effect of rarefaction wave along $\vec{\tau}_{1}$ is the main reason of pressure decreasing at point $O$.

(2) At point $A$, the rarefaction wave along $\vec{\tau}_{2}$ and $\vec{\tau}_{1}$ develop simultaneously.

(3) At point $A_{1}$, the rarefaction wave along $\vec{\tau}_{2}$ arrives at it earlier than that along $\vec{\tau}_{1}$. Therefore the effect of rarefaction wave along $\vec{\tau}_{2}$ is the main reason of pressure decreasing at

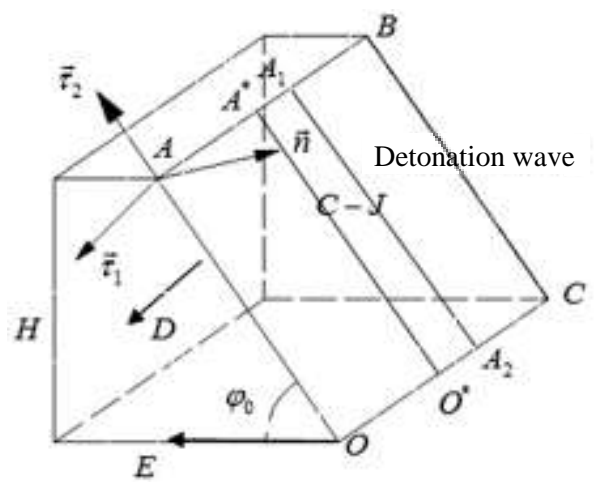

Fig.4 Scattering model of explosive detonation products 
point $A_{1}$.

(4) At point $A_{2}$, different from point $A$, there is a duration before the rarefaction wave arriving along both two tangential direction. If the rarefaction wave of $\vec{\tau}_{2}$ arrives earlier than that of $\vec{\tau}_{1}$, point $A_{2}$ can be considered as a point of center region; otherwise, $A_{2}$ is in periphery.

Thus demarcation between the periphery and the central region where the rarefaction wave along $\vec{\tau}_{2}$ and $\vec{\tau}_{1}$ arrives at the same time is line $O^{*} A^{*}$ as shown in Fig.4.

The average value of impulse in periphery can be obtained based on equation (3) by integration the impulse over $O O^{*}$ :

$$
\begin{aligned}
\bar{I}_{d O 0^{*}} & =\frac{\int_{0}^{l} o^{*} \int_{0}^{l / q_{\tau}} p(\tau) \mathrm{d} \tau \mathrm{d} l}{l_{o o^{*}}}=\frac{\int_{0}^{l} o^{*} \int_{0}^{l / q_{\tau}} p_{\max } \mathrm{e}^{(\tau / \theta)} \mathrm{d} \tau \mathrm{d} l}{l_{o o^{*}}} \\
& =\frac{p_{\max } \theta}{l_{O 0^{*}}}\left[q_{\tau} \theta\left(\mathrm{e}^{l o o^{*} l^{\left(q_{\tau}, \theta\right)}}-1\right)-l_{o 0^{*}}\right]
\end{aligned}
$$

Average impulse in central region:

$$
\begin{aligned}
I_{d O^{*} \rightarrow} & =\int_{0}^{\tau_{\mathrm{w}}} p(\tau) \mathrm{d} \tau=\int_{0}^{\tau_{\mathrm{w}}} p_{\max } \mathrm{e}^{(\tau / \theta)} \mathrm{d} \tau \\
& =p_{\max } \theta\left(\mathrm{e}^{\tau_{\mathrm{w}} / \theta}-1\right)
\end{aligned}
$$

Because of $\tau_{\mathrm{w}}=l_{o o^{*}} / q_{\tau}$ and let $\tau_{\mathrm{w}} / \theta=\eta$, the ratio of impulse in periphery to that in the central area is:

$$
Q=\frac{\bar{I}_{d o o^{\circ}}}{I_{d 0^{\circ} \rightarrow}}=\frac{\theta}{\tau_{\mathrm{w}}}-\frac{1}{\mathrm{e}^{\tau_{\mathrm{v}} / \theta}-1}=\frac{1}{\eta}-\frac{1}{\mathrm{e}^{\eta}-1}=\frac{\mathrm{e}^{\eta}-1-\eta}{\left(\mathrm{e}^{\eta}-1\right) \eta}
$$

Let's keep three terms in the series expansion, then:

$$
Q=\frac{\eta^{2}}{2 \eta^{2}+\eta^{3}}<\frac{1}{2}
$$

From the equation (6), we know that the ratio of average impulse in periphery to that in central region is less than 0.5. The impulse difference between in periphery and in central area can account for welding interface structure and vice versa. However, we have only achieved qualitative analysis for interface structure difference till now. Quantitative measurement which is the aim of this paper has not been achieved. The next section is the work we developed to try to describe the welding interface difference quantitatively.

Remarkable differences of interface morphology between the central region and periphery can be found from Fig.3. For the sake of fractal analyzing, these two images were processed to obtain binary images which are needed to calculate their fractal characteristics, and then fractal dimensions were calculated by a box counting method.

Box counting method is based on the calculation of number of squares with varying size of $\varepsilon$ of the image. The specific approach is ${ }^{[12,13]}$ : let the curve $F \subset R^{n}$ subset, $N_{\varepsilon}(F)$ is the minimum number of boxes of size $\varepsilon(\varepsilon<1)$ that can cover curve $F$, the definition of $D(F)$ is (when the limit exists):

$$
D(F)=\lim _{\varepsilon \rightarrow 0} \frac{\ln N_{\varepsilon}(F)}{-\ln \varepsilon}
$$

$D(F)$ is the box dimension of curve $F$. The probability distribution $p_{i j}(\varepsilon)$ of the photo pixel in surface image can be counted by the box dimension analysis method:

$$
\begin{aligned}
& P_{i j}(\varepsilon)=\varepsilon^{\alpha} \\
& N_{\alpha}(\varepsilon)=\varepsilon^{-f(\alpha)}
\end{aligned}
$$

where $\alpha$ is the singularity of the subset of probabilities which represents the inhomogeneity of probability distribution, $N_{a}(\varepsilon)$ is the number of boxes of size $\varepsilon$ with the same probability, and $f(\alpha)$ is the fractal dimension of the $\alpha$ subset.

We also calculated the weighted sum of the probability with an exponent $q$, and obtained the partition function $\chi_{q}(\varepsilon)$ :

$$
\chi_{q}(\varepsilon) \equiv \sum p_{i}(\varepsilon)^{q}=\varepsilon^{\tau(q)}
$$

The partition function $\chi_{q}(\varepsilon)$ was expressed as a power function of $\varepsilon$, and then the mass index expression $\tau(q)=\left(\ln \chi_{q}(\varepsilon)\right) / \ln \varepsilon$ can be obtained by the slope of curve $\ln \chi_{q} \sim \ln \varepsilon . \quad \tau(q)$ satisfies the expression $\tau(q)=\alpha q-f(\alpha)$, and the expression of $\alpha=\mathrm{d} \tau(q) / \mathrm{d} q$ can gain the fractal dimension $f(\alpha)$. A multi-fractal spectra curve can be plotted as $f(\alpha) \sim \alpha$ in which the metal surface undulations can be quantitatively characterized by horizontal width $\Delta \alpha=\alpha_{\max }-\alpha_{\min }$ of the curve and the ratio of the number of maximum and minimum subset of surface height can be revealed by the probabilities differences in ordinate $\Delta f=f\left(\alpha_{\max }\right)-f\left(\alpha_{\min }\right)$. Therefore morphology characters of the welding interfaces can be realized by the multi-fractal spectra curve ${ }^{[14]}$. According to the theory that has been mentioned above, fractal dimensions and multi-fractal spectrums of welding interfaces were calculated, shown in Fig.5 and Fig.6 respectively.

\section{Discussion}

The fractal dimensions of center region and periphery interface are calculated as $D_{1}=1.8329, D_{2}=1.7105$, respectively, as shown in Fig.5. The irregularities of geometry can be estimated by the physical meaning of the fractal dimension. The surface fractal dimension is closer to 2 with more complex interface. Thereby, $D_{1}>D_{2}$ means that complex surface structure in central region is more than that in the boundary, or that the center interface has finer microscopic structure.

The parameter $\alpha$ is the singularity of the probabilities subset. Because of $\varepsilon<1, \alpha_{\min }$ represents the maximum probability $\left(p_{\max }\right)$, while $\alpha_{\max }$ represents the minimum probability $\left(p_{\min }\right)$, and accordingly $\Delta \alpha$ represents the difference between the maximum and minimum probability $\left(p_{\max } / p_{\min }\right)$. Larger $\Delta \alpha$ means the more complex interface structure and represents wider distribution probability of the interface. In Fig.6, $\Delta \alpha_{1}=$ $\alpha_{\max 1}{ }^{-} \alpha_{\min 1}=0.41$ of central area and $\Delta \alpha_{2}=0.27$ of periphery. $\Delta \alpha_{1}>\Delta \alpha_{2}$ means interface roughness of the Fig.3a is larger than 


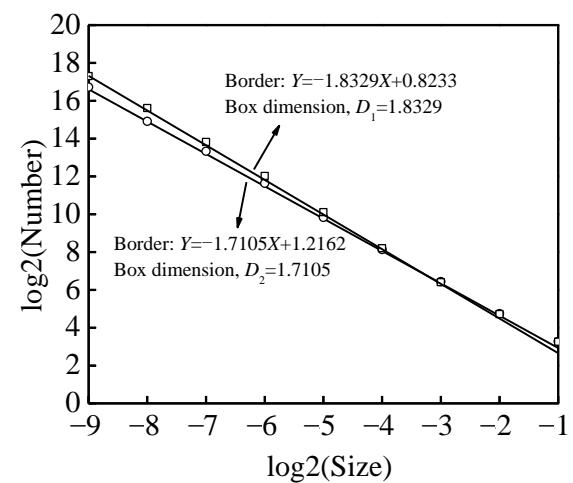

Fig.5 Fractal dimensions of central and periphery in EXW interface

that of the Fig.3b. On the basis of $\Delta \alpha$ physics meaning, we can come to the conclusion that the parameter $\Delta \alpha$ can achieve quantitative characterization for welding interface and it can replace the concepts of large wave, small wave and microwave in current literatures ${ }^{[2-5]}$ which all describe the interface topography qualitatively.

Parameters of the multi-fractal spectra can be used to characterize the shape of peaks and valleys of rough surfaces too. $f\left(\alpha_{\min }\right)$ represents the number of boxes of the maximum probability, while $f\left(\alpha_{\max }\right)$ reflects the number of box of minimum probability ${ }^{[10]}$. Thus, $\Delta f$ automatically represents the ratio of the number of sets of the peaks to that of the valleys statistically. In Fig.6, because of $\varepsilon<1, \Delta f=f\left(\alpha_{\max }\right)-f\left(\alpha_{\min }\right)>0$ means the number of maximum probability subset less than that of minimum one, and demonstrates the relatively sharp valleys and peaks.

Based on the entropy theory, we know that the fractal dimension calculated from the number of box gets the information about the fractal character of the image of welding interface. Thus it can be also regarded as information dimension. Then according to the dependence between the Shannon entropy and the information dimension ${ }^{[15]}$, the Shannon entropy can be written:

$$
S_{\text {Shannon }}=D \ln \frac{1}{\varepsilon}
$$

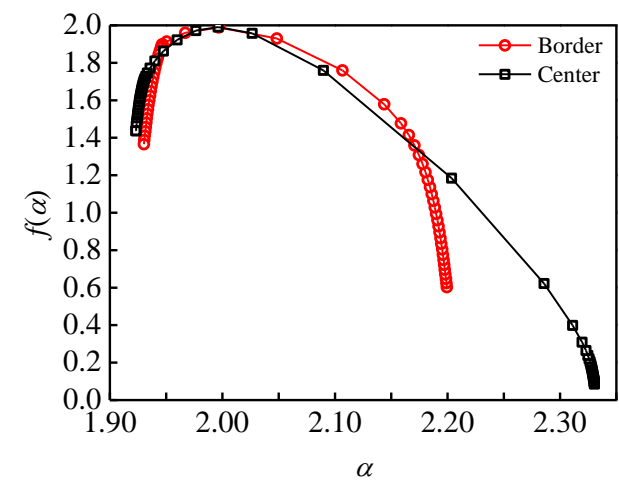

Fig.6 Multi-fractal spectrum of welding interface
Furthermore, the thermodynamic entropy $S$ can be obtained by multiplying Shannon entropy by Boltzmann constant $k_{B}$ :

$$
S=k_{\mathrm{B}} S_{\text {Shannon }}=k_{\mathrm{B}} D \ln \frac{1}{\varepsilon}
$$

The entropy $S$ introduced in the second law of thermodynamics is easy to understand for us. Therefore, by Eq.(13), we can know the ratio of the thermodynamic entropy of central region to that of periphery by the fractal dimension in Fig.5:

$$
\frac{S_{1}}{S_{2}}=\frac{D_{1}}{D_{2}}=\frac{1.8329}{1.7105}
$$

According to the second law of thermodynamic, we know that the thermodynamic entropy has relationship with energy. Thus, the energy state difference between the central region and periphery can be even evaluated by the fractal analysis.

Thereby, fractal dimensions of contour line in Fig.5 reflect the physical complexity of interfaces, and multi-fractal spectrums in Fig.6 reveal significant geometry differences of the uniformity in welding interfaces which are mainly caused by the detonation pressure fluctuation.

\section{Conclusions}

1) By observing the contour lines and the calculation of interfaces fractal dimensions, it can be concluded that the welding interface in this paper has fractal characteristics. This demonstrates that the interface has more complex structure. So it's insufficient to describe the interface structure only by the magnitude of wave.

2) The fractal dimension in central region is slightly different from that of the periphery. Because of the effect of detonation rarefaction waves, the fractal dimension and the complexity in periphery is a little less than that in the central region.

\section{References}

1 Zhang Y, Babu S S, Prothe C et al. Journal of Materials Science \& Technology[J], 2011, 211(5): 944

2 Chizari M, Al-Hassani S T S, Barrett L M. Journal of Materials Processing Technology[J], 2009, 209(1): 445

3 Akbari-Mousavi S A A, Barrett L M, Al-Hassani S T S. Journal of Materials Processing Technology[J], 2008, 202(1-3): 224

4 Drennov O B, Davydov A I, Mikhailov A L et al. International Journal of Impact Engineering[J], 2005, 32(1-4): 155

5 Fehim F. Materials and Design[J], 2011, 32(3): 1081

6 Drennov O B, Mikhailov A L, Nizovtsev P N et al. International Journal of Impact Engineering [J], 2005, 32(1-4): 161

7 Ben-Artzy A, Stern A, Frage N et al. International Journal of Impact Engineering [J], 2010, 37(4): 397

8 Bellman R, Pennigton R H. Quarterly of Applied Mathematics[J], 1954, 12(2): 151

9 Lee H G, Kim J. European Journal of Mechanics-B/Fluids[J], 2015, 49: 77 
10 Mandelbrot B B. The Fractal Geometry of Nature[M]. NewYork: WH. Freeman and Co, 1982

11 Lysak V I., Kuzmin S V. Journal of Materials Processing Technology[J], 2012, 212(1): 150

12 Sun X, Fu Z X, Wu Z Q. Physica A: Statistical Mechanics and Its Applications[J], 2002, 311(3): 327

13 Chen Z W, Lai J K L, Shek C H. Physics Letters A[J], 2005,
345(1-3): 218

14 Sun X, Wu Z Q, Huang Y. The Principle and Applications of Fractal Theory[M]. Hefei: University of Science and Technology of China Press, 2003 (in Chinese)

15 Oldrich Z, Petr D, Michal V. Computers and Mathematics with Applications[J], 2013, 66: 135

\title{
Al-Al 爆炸焊接界面边侧与中心区域差异定量评价
}

\author{
付艳恕, 夏 萌, 王 震 \\ (南昌大学, 江西 南昌 330031)
}

\begin{abstract}
摘 要: 由于边侧稀疏波的作用, 爆炸焊接界面边界与中心区域形貌结构存在差异。然而学术上对焊接界面形貌差异分析仍处于定性水 平, 尚未建立定量分析方法。据此, 尝试对爆炸焊接界面形貌差异开展定量化评价。研究过程中首先建立冲击波作用下金属复板弹粘塑 性模型以分析波状界面的形成, 并开展 2024Al 对称爆炸焊接; 随后运用三维超景深显微镜获得界面形貌图像, 结合冲量理论, 定义中 心区域与边侧区域分界线; 最后基于分形理论计算图像轮廓分维与多重分维谱。由分维与多重分形谱数据可定量表征界面的起伏程度与 表面最大、最小概率分布, 从而实现焊接界面形貌差异定量描述。
\end{abstract}

关键词：爆炸焊接；界面形貌；分维；表征

作者简介: 付艳恕, 男, 1982 年生, 博士, 副教授, 南昌大学机电工程学院, 江西 南昌 330031, 电话: 0791-83969635, E-mail: yshfu@ncu.edu.cn 